\title{
Benateyê Mewlidê Mela Kamilê Pueği u Mela Mehamedê Muradoni Dı Yo Muqayese
}

\section{Molla Kamil Pueği ile Molla Muhammed Muradoni’nin Mevlitlerine Dair Bir Karşılaş̧ırma \\ A Comparison of the Mewlids of Mullah Kamilê Pueği and Mullah Mehamedê Muradoni}

\author{
Murat Varol ${ }^{\mathrm{a}, *}$ \\ ${ }^{a}$ Öğr. Gör., Bingöl Üniversitesi, Fen Edebiyat Fakültesi, Doğu Dilleri ve Edebiyatı Bölümü, 12000, Bingöl/Türkiye. \\ ORCID: 0000-0002-1706-9990
}

\section{MAKALE BILGIISI}

\section{Makale Geçmişi:}

Başvuru tarihi: 27 Ocak 2017

Düzeltme tarihi: 31 Mayıs 2017

Kabul tarihi: 18 Temmuz 2017

Anahtar Kelimeler:

Klasik Edebiyat

Molla Kamil Pueği

Molla Muhammed Muradoni

Zazaca

\section{ARTICLE INFO}

\section{Article history:}

Received 27 January 2017

Received in revised form 31 May 2017

Accepted 18 July 2017

\section{Keywords:}

The Classical Literatüre

Mullah Kamil Pueği

Mullah Mehamedê Muradoni

Zazaki

\section{ÖZ}

Zaza edebiyatı, 1899 yılında Molla Ahmed-i Xasi'nin yazdığı mevlit ile başlamıştır. Ondan sonra bir başka molla Osman Efendi de bir mevlit yazmıştır ve bu da Zazaca'nın ikinci eseridir. Bu iki molladan sonra Molla Muhammed Muradoni, Molla Kamil Pueği, Molla Muhammed Ali Huni, Molla Abdulkadir Muşeki, Bilal-Feqi Çolig, Mehmet Akif Demir mevlit yazmışlardır. Zaza edebiyatında toplam sekiz mevlit eseri bulunmaktadır. Bu makalede, Molla Kamil Pueği ile Molla Muhammed Muradoni'nin mevlidi mukayese edilecektir. Her iki mollanın hayatına, medrese eğitimine yer verilecek, sonrasında da her iki mevlit; vezin, kafiye ve mevlidin muhtevası bakımından incelenecektir.

\section{A B S TR ACT}

Written literature of Zazaki started in 1899 with the mewlid of Ehmedê Xasi. After Xasi, another mullah-Osman Efendiyo Babij wrote the second study and it was a mewlid as well. These two studies are both the examples of the classical literature. The written literature of Zazaki has continued with other writers. After these two writers, Mullah Mehamedê Muradoni, Mullah Kamilê Pueği, Mullah Mehamedê Eli Huni, Mullah Ebdulqadirê Muşeki, Bilal-Feqi Çolid, Mehmet Akif Demir others wrote literary studies in the style of classical literature. In this study, I will compare the mewlids of Mullah Kamilê Pueği and Mullah Mehamedê Muradoni. Their educational phases, the writing time of their mewlids, the similarities, rhythym and rhyming styles will be compared.
\end{abstract}

\section{Xulasa}

Zazaki serra 1899 dl bl Ehmedê Xasi destpê kerd u ey mewlidêk nuşto. Xasi ra dıma yona mela, Osman Efendiyê Babiji kıtabê diyın nuşt u o zi mewlid o. İnan ra dima Mela Mehamedê Muradoni, Mela Kamilê Pueği, Mela Mehamedê Eli Huni, Mela Ebdulqadırê Muşeki, Bilal-Feqi Çolig, Mehmet Akif Demiri mewlidi nuşti. Edebiyatê Zazaki dı heşt mewlidi nusiyayi. Ina meqale dl mewlidê Mela Kamilê Pueği u Mela Mehamedê Muradoni muqayese beni. Verco ca diyeno heyatê nuştoğan, dıma zi wırdi mewlidi goreyê wezin, qafiye u muhtewa tetkik beni.
Çekuyi Muhimi: Edebiyato klasik, Mela Kamilê Pueği, Mela Mehamedê Muradoni, Zazaki

\section{Destpêk}

Kelimeya mewlid, Erebki ya u rıstımê kelimeya weladet ra yena, zafiyê ına kelime zi mewalid a. Bi manaya xısusi ına kelime, kıtabi ki biyayiş u cuyayişê Hz. Pêxımberi ra behs keni qê inan yena şıxulnayiş. Mewlidan di teyna biyayiş $\mathrm{u}$ cuyayişê Hz. Pêxımberi nê, bê ıneyi hediseya miraç ra heta mucizeyanê Hz. Pêxımberi u wefatê ey, zaf çi ra behs beno. Kitabi mewlid verco teyna qê Hz. Pêxımberi nusiyayi, labelê

* Sorumlu yazar/Corresponding author.

e-posta: mvarol@bingol.edu.tr 
wextan ra pê hêdi bı hêdi qê cuyayişê weliyan u şexsiyetanê pilan zi mewlidi ameyi nuştış (Özel, 2004: 475). Mewlidi bı zafi manzum i u şıklê mesnewi di yeni nuştış (Pala, 1999: 275). Nuşteyi mewlidi, miyanê şari dı şewa biyayişê Hz. Pêxımberi dı waniyeni. Bê ıneyi bı zafi qê çiyêko bımbarek u şayi zi mewlid diyeno wendış.

\section{Tarıxê Edebiyatê Zazaki ê Klasiki}

Edebiyato klasik, edebiyatanê miletan d1 cayêko muhim depışeno. Alımo ki tehsilê medresa diyo u icazet gıroto, eşqê Homayi u Pêxımberan pê nuşteyanê klasikan ano zıwan. Edebiyatê Zazaki ê klasiki dı teyna hirı şıklan d1 kıtabi nusiyayi. Ineyi zi mewlid, siyer u diwan $i$.

Edebiyatê Zazaki pê nuşteyanê klasikan destpê kerdo. Dora vêrin Mela Ehmedê Xasi serra 1892 di mewlidêk nuşto u ino mewlid serra 1899 dı Diyarbekir dı Matbaaya Litografya dı 400 heb neşır biyo. Mewlidê Mela Ehmedê Xasi heta eyro çend dori bı herfanê Latini transkribe biyo. Xasi ra pê kıtabê dıyın ê Osman Efendiyê Babıji yo. Goreyê zanayişan Osman Efendi mewlidê xu serra 1903 di nuşto; labelê ıno mewlid, mergê Babıji ra dıma serra 1933 di dora vêrin miyanê kıtabanê Hawari ra vêjiyayo. Ini wırdi mewlidan ra pê nuşteyi Zazaki wextêko derg vınderti. 1970 ra pê seydayanê medresa nuşteyanê klasikan rê dewam kerdo. Mela Mehamedê Eli Huni, Mela Kamilê Pueği, Mela Mehamedê Muradoni, Mela Ebdulqadırê Muşeki, Mela Eziz Cebexçuri inan ra çend ten $i$.

Mela Mehamedê Eli Huni, mewlidê xu serra 1971 dı nuşto; labelê mewlid 33 serri cuapê serra 2004 dı neşir biyo. Kıtabê Mela Huni dı pa mewlidi ra, dı zi qesideyi ca geni. Qesideya vêrin di Yusuf u Zuleyxa ra ê dıyın dı zi hediseya Kerbela ra behs beno. Mela Mehamedê Muradoni mewlidê xu serra $1980 d_{1}$ dora vêrin qeyd keno bandan. Dıma zi serra $2000 d_{1}$ 1ni bandan ra mewlidê xu nuseno u keno temam. Serra 2003 dı zi kovara Vate di neşır beno. Mewlidê pancın zi ê Mela Kamilê Pueği yo u serra 1999 di nusiyayo, serra 2003 di İstanbol dı neşır biyo. Mela Ebdulqadırê Muşeki, mewlidê xu serra 1995 d 1 nuşto, 1no mewlid dora verin serra 2016 d 1 transkribe bı u mecmuaya Vir dı neşır biyo. Feqi Çolig zi qê tutan mewlidêk nuşto u serra 2012 di dayo çapkerdış. Mewlido peyin zi Mehmet Akif Demir serra 2017 dı neşır kerdo.

Bê inan çendna kıtabi edebiyatê Zazaki ê klasiki esti. Zazaki dı heta eyro teyna yo kıtabê siyeri nusiyayo u muellifê $\mathrm{c} 1 \mathrm{zi}$ Milla Cimayo Babiji yo. Milla Babiji kitabê xu serra 2009 di neşır kerdo. Edebiyatê Zazaki dı heta eyro çend hebi zi diwani neşır biyi. Diwanê Mela Mehamedê Kavari inan ra yo. Diwanê Mela Kavari, 70 rıpeli herfanê Erebki u 70 rıpeli zi herfanê Latini pêser di 140 rıpeli ra yeno meydan. Edebiyatê Zazaki ê klasiki dı kıtabanê tor muhiman ra yo zi ê Mela Aziz Cebexçuri yo. Seydayê erciyayi Mela Aziz, diwanê xu serra 2015 di binê mahiyetê neşirxaneyê universiteya Bingoli dı veto. Diwan, benateyê serranê 2005 u 2015 di nusiyayo, 435 ripeli ra yeno meydan u him b1 herfanê Erebki u hım zi herfanê Latinki neşır biyo. Edebiyatê Zazaki dı miyanê nuşteyanê klasikan dı kıtabi tercume zi esti. Heta eyro di diwani tercume biyi Zazaki. Tercumeyê wırdi diwanan zi heti Feqi Çoligi ra vıraziyayo. Ini kitabi zi Diwanê İmamê Şafii u Diwanê Baba Tahirê Uryani yi. Bê inan zi kıtab u nuşteyi klasiki esti.
B1 kılmı heta eyro edebiyatê Zazaki ê klasiki dı heşt mewlidi, yo siyer, çend diwani, di diwani tercume u ge ge zi mecmuayan di çend şiiri, qesideyi u nuşteyi muxtelif neşır biyi. Edebiyatê Zazaki ê klasiki di çend destnuşteyi ya zi kıtabi zi esti ki hema neşır nêbiyi. Ina meqale dı teyna mewlidê Mela Kamilê Pueği u Mela Mehamedê Muradoni geriyeno dest.

\section{Heyatê Mela Kamilê Pueği u Mela Mehamedê Muradoni}

Mela Kamilê Pueği, serra 1938 dı dewa Çolig Pueğ dı ameyo dinya. Q1ctiya ey dew di viyert ra. Verco dest bi wendışê Qur'an kerd. Az u Sıpeni dı wendışi rê dewam kerd u Sıpeni dı şeş serri tehsilê medresa di. Etiya dı Seyda Mela Suleymani ra dersi sarf u nahif gıroti. Dewanê Ğezık u Pueğ dı hirı serri dersi mantıq u e qaidi Seyda Mela Mehmudi ra gıroti. İnan ra pê şı esker u qezaya Wan Erciş d 1 30 aşmi eskerayi kerd. Eskerayi ra pê tehsil rê dewam kerd. Medresayanê Diyarbekır u Mardin dı dersi tefsir, fiqıh u hedisi giroti. Wext 27 serran di b1, Mela Mehmudi ra icazet girot u tehsilê medresa kerd temam. 2 serri imamtiya fexri kerd u dıma zi serra 1966 di dest bı imamtiya resmi kerd, dewanê Duedon u Rotcun di heta bi teqawit melati kerd. Teqawıti ra pê demêk tucaret $d_{1}$ muştığ 1 l bı. Inka Çolig $d_{1}$ cuyeno u bınê Camiya Heci Xıdır dı cayêkê ey esto. Etiya dı b1 usılê medresa ders dano. ${ }^{1}$

Mela Mehamedê Muradoni, serra 1952 di qezeya Çolig Darêyeni, dewa Muradoni dı ameyo dinya. Tehsilê xu yo vêrin, babiyê xu ra girot. Dıma tehsilê xu rê Muş dı dewam kerd. Mela Mehamedi, 1976 dı qezeya Çolig Çêrme dı destpê kerd wezifeyê xu u etiya dı yo serr u nim xebıtiya. Dıma zi qezaya Çolig Boglon dı hirı serri xebıtiya. İxtilalê 1980 ra pê surgın bı qezaya Çoligi Geğı. O zi istifaya xu dano u şıno Mardin Kızıltepe, etiya dı medresaya Şêx Selahedini dı d1hirı serri ders dano. Cuapê istifaya xu têpiya geno u tayin beno şarıstanê Çorum. Çorum di teyna yo serr maneno $u$ ageyreno yeno Palı. Etiya di yo medresa virazeno u panc serri ders dano. Dima zi qerarê surgun dariyeno we $u$ ageyreno Darêyeni u etiya dı wezifeyê xu rê dewam keno (Canşad, 2011). Seyda imamti ra biyo teqawıt u ınka zi Bursa di cuyeno.

\section{Wextê Nuştışê Mewlidê Mela Kamilê Pueği u Mela Mehamedê Muradoni}

Mewlidê Mela Kamilê Pueği, serra 1999 dı nusiyo; labelê serra 2003 d İstanbol d neşır biyo. Mewlidê Mela Kamil d 1 bê mewlidi, 8 hebi şiiri zi ca geni. Mewlid, 104 beyitan ra yeno meydan u W.K. Merdimîn transkribe kerdo. Ino mewlid, b1 nameyê Mewlidi Nebi yeno zanayiş. Derheqê Mela Kamilê Pueği di kovara Vate omaranê 171n, 18ın u 191n d 1 çend nuşteyan ca gıroto. W.K. Merdimîn, mecmuaya Vate omara 17ın di ca dayo 2 şiiranê Mela Pueği. Ini şiiri b1 nameyê Welatê Ma u Merg neşır biyi (Merdimîn, 2002). Heyna kovara Vate omara $181 n$ di Mela Kamilê Pueği d 1 roportajêk viraziyayo u ino roportaj di Mela Kamil derheqê xu u nuştışe xu yê Zazaki dı melumat dano (Merdimîn, 2002). Omara 191n di zi mewlidê ey ına mecmua dı neşır biyo (Pueği, 2003). Ino mewlid 104 beyitan ra yeno meydan u mewlid di qisımi ca nêgeni, mewlid sare ra heta peyni yo lete yo. 
Mela Mehamedê Muradoni wexta ki hema Mardin Kizıltepe dı bı, şiiri Zazaki nuşteni. Hım şiiranê xu u hım zi mewlidê xu qêyd keno bandan. 1999 di mewlidê xu inê bandan ra nuseno u keno temam. Mewlidê Mela Mehamedê Muradoni, serra 2003 dı kovara Vate dı neşır beno (Muradon, 2003). Ina omar $\mathrm{d}_{1}$ bê mewlidi yo zi roportaj ca geno $\mathrm{u}$ ino roportaj $\mathrm{d}_{1}$ Mela Muradoni derheqê xu, nuştışê xu yê Zazaki u mewlidê xu di melumat dano (Merdimîn u Celalî, 2003). Mewlid heti W.K.Merdimîn u N.Celalî ra ameyo transkribekerdış. Ino mewlid, 7 q1sıman ra yeno meydan u mewlid di 204 beyiti esti. Mewlidê Mela Mehamedê Muradoni, bi nameyê Mewlidi Zazaki ameyo namekerdış.

\section{Tehlilê Mewlidi Mela Kamilê Pueği u Mela Mehamedê Muradoni}

Mewlidi Mela Kamilê Pueği u Mela Mehamedê Muradoni zaf cihetan ra maneni yobinan. Ma 1 no qisım dı mewlidanê Mela Kamili u Mela Mehamedi heti uslub, wezin, qafiye u muhtewayê mewlidi ra tetkik keni. Ma ına meqale dı qê tetkikê mewlidan, nusxayi ki kovara Vate dı neşır biyi, ıni esas giroti.

\subsection{Uslub u Ziwanê Mewlidan}

Mela Kamilê Pueği u Mela Mehamedê Muradoni, Çoligij i. Wirdi zi bı Zazakiya merkez nuseni. Zıwan u uslubê wirdi seydayan di fekê Çoligi xu mojneno ra. Nuştışê çekuyan, weziyetê izafeyan, zafiyê kelimeyan, nuştışe karan u zaf çiyi bin heme maneni yobinan. Yona xisusiyetê wirdi seydayan, bı zıwanêko fesih u zılal mewlidi xu nuşti. Mewlidanê inan dı Erebki, Farıski u zıwananê binan ra zaf kelimeyi çin i.

\subsection{Weznê Mewlidan}

Nuşteyi edebiyatê klasiki bı weznê aruz nusiyeni. Mewlidê Mela Kamilê Pueği u Mela Mehamedê Muradoni zi bı weznê aruz nusiyayi. Labelê wirdi seydayan zi weznê aruz xerıpnayi şıxulnayo. Qê wezin zafi beyitan dı heceya akerde u heceya qefilnaye rê diqet nêbiyo. Wirdi seydayan zi bi zafi teyna aheng u qafiyeya beyitan rê diqet kerdo. Him mewlidê Mela Kamilê Pueği di hım zi mewlidê Mela Mehamedê Muradoni dı qusırê aruzi esti.

Wirdi mewlidan dı qalıbê Fâilâtun/Fâilâtun/Fâilun ameyo şıxulnayiş. Mewlidê Mela Kamilê Pueği dı beyita hirıyın goreyê aruz ma eşkeni ina tehlil bıker.

\section{Allah zat û / sifetûn xwi d' / yo ten û}

- - - / - . - - / - -

Bê yi çewnê / Reb nêken nê / vînenû

- . - - / . - - / - -

Fâ/i/lâ/tun / Fâ/i/lâ/tun / Fâ/i/lun

Mewlidê Mela Mehamedê Muradoni dı zi ma eşkeni qê aruzi bıoni beyita dıyın a. Labelê ına beyit dı zi qusırê aruzi ca geni.

Ma pê yi dest / kerdi mewli / dî nebî

- . - - I . . - / - . -

Ay hebîbî / ay tebîbî / ay ummî.

- . - - / - - - / - .

Fâ/i/lâ/tun / Fâ/i/lâ/tun / Fâ/i/lun

\subsection{Qafiyeya Mewlidan}

Qafiyeya nuşteyanê klasikan d1, bı zafi dı usuli esti. Ya her beyit benateyê $\mathrm{xu}$ di qafiyeyın a ya zi beyita vêrin benateyê xu dı qafiyeyın a, beyitanê binan dı zi rêzi vêrin xuser $i$, rêzi dıyın zi beyita vêrin dı qafiye ani meydan. Mewlidi bı terzê mesnewi $\mathrm{d}_{1}$ yeni nuştış, usulê mesnewiyan $\mathrm{d}_{1}$ her beyit benateyê xu di qafiyeyın a.

Kitabê Mela Kamilê Pueği Mewlidê Nebi u kitabê Mela Mehamedê Muradoni Mewlidê Zazaki, hetê rêzkerdışê qafiye ra eyni yi. Wirdi mewlidan dı zi her beyit benateyê xu di qafiyeyin a.

\section{Mewlidê Mela Kamilê Pueği}

Pey Allay Mewlîd Nebî xwi r' wûneno a

Allah wahar canûn û camîdûn o a

Weqta g' Allay waşti g' mexluq yi vînû b

Pey emrî "kûn" 'erd û ezmûn ard meydû b

Allah zat û sifetûn xwi d' yo ten û

Bê yi çewnê Reb nêken, nêvînenû

\section{Mewlidê Melamedê Muradoni}

Allah, nûme xaliqî mawo 'elîm, Hem 'ezîm û hem kerîm û hem rehîm.

Ma pê yi dest kerdi mewlidî nebî, a Ay hebîbî, ay tebîbî, ay ummî.

Hemd û şukr û medh û fexrî tim dewam Rebbî ma rî zu'l-celalî bî’t-temam.

\section{Muhtewaya Mewlidan}

B1 tabloyêko umumi ma eşkeni vaji ki yo mewlidi d1; q1sımi vıraştışê kainati, xelqbiyayişê Hz. Ademi, nurê Pêxımberti, b1 silsile viyertışê ınê nuri, şewa weladeti, mu'cizeyi şewa biyayişê Hz. Pêxımberi, şewa Mirac u du'a ca geni. Tayn mewlidan dı heme 1 ni q1sımi ca geni, tayn mewlidan dı zi ınê q1sımi beni ziyed ya zi beni kêm. Misal tayn mewlidanê Zazaki dı şewa Miraci çin a, tayn mewlidi zi teyna heyatê Pêxımberi ra çend merhelayan nê, hemeyi heyatê Pêxımberi ra behs keni.

\subsubsection{Qısımê Hemd u Şıkır}

Mewlidi Mela Kamilê Pueği u Mela Mehamedê Muradoni, heti muhtewa ra zaf maneni yobinan. Mewlidi Mela Kamilê Pueği u Mela Mehamedê Muradoni, pê hemd u şıkır destpê keni. Ino qisım, wirdi mewlidan dı zi destpêk hesıbiyeno. Hım mewlidê Mela Kamilê Pueği dı hım zi mewlidê Mela Mehamedê Muradoni di 17 beyitanê vêrinan dı hemd u şıkır ca geno.

\section{Mewlidê Mela Kamilê Pueği}

Hemd û şikir Allay xwi r' dewam kirîn Şewûn, ruejûn, tım vajîn, ter mekirîn

Bûn' dinya ra gêyrena tadîyena Qey xatîr tu nî‘îmetûn bedilnena

Ey Muhemmed, ey ti enwaru'l-huda! Hem sened û mesnedî kurey dinya!

Mewlidê Mela Mehamedê Muradoni

Hemd û şukr û medh fexrî tim dewam 
Rebbî ma rî zu'l-celalî bî’t-temam

Yi ma kerdî ummetê Ehmed' hebîb

Ay nebîyî, ay resûlî, ay tebîb

O resûl ku serwerî pîyor enbîya

Dilberî ay ewlîya û esfîya

\subsubsection{Qısımê Nurê Pêxımberti u Merheleyi Ino Nuri}

Qisımê diyın dı wirdi mewlidan dı zi nurê pêxımberti u ino nur Hz. Adem ra heta Hz. Muhammed (s.a.w.) seni ameyo, ıneyi ra behs beno.

\section{Mewlidê Mela Kamilê Pueği}

Va Cibrîl ra: Bigeyr 'erd û ezmûnûn d'

Hem behrûn û şerq û xerbê etrafûn d'

Ûme geyra, behs' Adêm qet çine yo

Dar, kerri pîyor vûn "Muhemmed nebî yo".

Adnan a cêr heta g' Ebdullay resû

Vîst û yew babî d' pa doxrû-durust û

\section{Mewlidê Mela Mehamedê Muradoni}

Bizûnê ewwelî mexlûqî îlah,

Nurî Peyxamberî ma w’ bêîştîbah.

Yi wa ver çine bi mexlûqêk ebed.

Xaliqî ma têna bi; wehîd, ehed.

Xelqi kerdi Ademî babî beşer.

Nûrî Peyxamber' resa yi mu'teber

Beyitan dı zi eseno ki muhtewaya ınê qısımi zêcê ya. Labelê ifadekerdışê ınê xısusi dı ferq esto. Wırdi mewlidan dı zi q1sımê hemd u şıkır ra pê qısımê vıraştışê kainati, xelqbiyayişê Hz. Ademi u nurê Pêxımberti Hz. Adem ra heta Hz. Pêxımberi seni ameyo, inan ra behs beno.

Mewlidê Mela Kamilê Pueği dı beyita 18 nn ra heta beyita 481n ino xısus esto. Yani 30 beyitan di Hz. Adem seni vıraziyo, nurê Pêxımberti seni derc biyo, Hz. Adem ra heta Hz. Pêxımberi ıno nur seni yo merhale ra viyerto ra, ınê X1susi ca geni.

Mewlidê Mela Mehamedê Muradoni dı zi qisımanê diyın u hirıyın dı eyni xısus ra behs beno. Inê xısusi zi zê Mewlidê Pueği yi. Mela Muradoni zi Hz. Adem ra geno heta $\mathrm{Hz}$. Pêxımberi, nurê Pêxımberti u merhaleyanê ınê nuri ra behs keno. Mela Muradoni goreyê Mela Pueği bınêna hira ca dano ıno qisım u 48 beyitan di inan ra behs keno.

\subsection{3. Şewa Biyayişê Hz. Pêxımberi}

Mewlidan dı qısımê hirın dı şewa biyayişê Hz. Pêxımberi ra behs beno. Şewa ki Hz. Pêxımber ameyo dınya, a şew dı se biyo, çı mu'cizeyi ameyi meydan ıneyi zi ca geni.

\section{Mewlidê Mela Kamilê Pueği}

Amîne va: Ez teyna menda îtya

Be cinyûn, cûmêrdûn tersû ez îtya.

Mi dîy car heb cinî ûmey cayê min

Zaf bedew b', rindîyê yin ruaşn da min.

Emir kerd, Cebraîl ra va: Bê îtya!

Hem mîjdun bid' pêro mexlûqê dinya.

\section{Mela Mehamedê Muradoni}

Mijdûnî dûn yobînî zewq û sefa, Qey ku yenû dunya Ehmed, Mustefa.

Vûna: Ez têna b’ya weqt' weladetî, Nê cûmêrd bi nê cinî ay sa'etî.

Mi dî çend cinî ke derg bî zafí-zaf,

Mi va qey yi neslê ay 'Ebdulmenaf.

Ini qısımi mewlidi maneni yobinan. Mewlidê Mela Kamilê Pueği dı şewa ki Hz. Pêxımber ameyo dınya, mijdanê ına şew dano heme mexluqatan. Dima zi Hz. Amine, a şew çı diyo inan ra behs beno. Ina şew di Hz. Amine teyna ya, labelê çend ciniyi yeni cayê ay u teyna nêverdeni, dıma zi Hz. Muhammed (s.a.w.) yeno dınya u a şew çı mu'cizeyi ki bi inan ra behs beno.

Mela Mehamedê Muradoni zi hema hema bı eyni xısusan ıno qisım nuseno. O zi verco mijdanê biyayişê Hz. Pêxımberi dano. Şewa ki Hz. Amine teyna ya o zi behs keno u ciniyi ki ameyi cayê Hz. Amine nameyê ini ceniyan dano. Peyniya qısımi dı zi wazeno ki her kes selat u selam biyaro.

\subsubsection{Qusımê Merheba}

Biyayişê Hz. Pêxımberi ra pê, qê ey qisımê selat u selam ca geno. Ino q1sım dı hım şayi esta u hım zi şefa'et waziyeno.

Q1sımê merheba, wırdi mewlidan dı manena yobinan. Wırdi mewlidan di zi peyniya beyita vêrin pê kelimeya merheba qêdiyena u dıma zi kelimeya merheba yena verniya beyitan.

\section{Mela Kamilê Pueği}

Qêy hîdayêt îns û cin yi g' şeref da

Pey veng berz pîyorîn va: Tu r' merheba!

Merheba, ti rehberê her kesûn $1 \hat{~}$

Merheba, ti pîyorê derdûn dermûn $\hat{i}$

Merheba, ti eşrefî̀ wir d' dinyûn î

Merheba, ti peyinî pêxmerûn $\hat{\imath}$

\section{Mela Mehamedê Muradoni}

Pîyorî 'alem pe ûmeyiş yi bi şa, Eşq û cezbe ra pîyorin va: Merheba.

Merheba, ey rûh û canî 'alemî.

Merheba, ey derdî eşqî r' melhem î.

Merheba, ey muqteday' ruejî qîyam.

Merheba, peyxamberûn rî ti îmam.

Ino qısım di wesıfanê Hz. Pêxımberi ra zi behs beno. Raybertiyê ey, serwertiyê hım ına dınya hım zi dınyaya bin, Pêxımberi axirzeman u çiyo bin ra behs beno. Etiya dı wırdi seydayi Hz. Pêxımberi ra şefa 'et zi wazeni u qayili ki gunayi inan ef bibi.

\subsubsection{Qısımê Dua}

Mewlidi Mela Kamilê Pueği u Mela Mehamedê Muradoni pê qısımê dua qêdiyeni. Wırdi melayi zi ıno qısım dı hım qê $\mathrm{xu}$ hım zi qê umet, Hz. Pêxımberi ra şefa'et wazeni. Ino q1sım di vaciyeno ki insani rehmê Homayi ra omıdê $\mathrm{xu}$ nêbırneni u qayili şiyeri cennet $u$ gunayan ra ef bıbi. 


\section{Mela Kamilê Pueği}

Umîdwarê rehm û îhsanî tu yî

Hem talibî 'ef û xufranî tu yî

Ya Reb! Ti vînenî, hem eşnawenî

Gunê ma hîsab nêbên, n'ûmaryenî

'Ef bide qey xatirî Muhemmedî

Ummetê yi şadan kir cennêt xwi di

\section{Mela Mehamedê Muradoni}

Ay resûl î, ay hebîb î, serwer $\hat{i}$,

Eşq û hubb' tu benû qelb ra kederî.

Wesfî tu zê miskî dûno buya weş,

Pe vatiş nîqedyeno pê pûnc û şeş.

Mu'cizêy tu zêdey' pîyorê enbîya.

Feyz û nûr gên tu ra pîyorî ewlîya.

Mela Kamilê Pueği q1sımê dua dı gunehkariyê insanan ra behs keno u qê inê gunayan Homayi ra ef wazeno. Mela Kamili, Hz. Adem seni nameyê Hz. Muhammedi (s.a.w.) dayo u ef biyo, o zi nameyê Pêxımberi dano u qê xatıri Pêxımberi ef wazeno. Mela Mehamedê Muradoni zi q1sımê dua dı xısusiyetanê Resuli ra behs keno u o zi peyniya qısımi dı ef wazeno. Ino qisım mewlidê Mela Mehamedê Muradoni dı derg o zê mewlidê Mela Kamilê Pueği yi.

\section{Netice}

Mela Kamilê Pueği u Mela Mehamedê Muradoni, edebiyatê Zazaki ê klasiki dı cayêko muhim depıșeni. Wirdi seydayi Çoligij i u tedrisatê medresa ra viyerti ra, wırdin zi melati kerda $\mathrm{u}$ biyi teqawıt. Wextê nuştışe wırdi mewlidan zi maneno yobinan. Wezin u qafiyeya mewlidan zi eyni ya. Heti qısımanê mewlidan ra, wirdi mewlidi zaf maneni yobinan. Mewlidê Mela Muradoni yo-dı qisımi derg o zê Mewlidê Pueği yi. Mewlidi bı hemd u şıkır destpê keni, bı nurê Pêxımberti dewam keni; dıma zi şewa weladeti, merheba $u$ dua yeno. Wırdi seydayan di no rêzkerdış esto, seydayan di teyna vatıs yani uslub bedıliyeno. Mewlidi Mela Kamilê Pueği u Mela Mehamedê Muradoni, miyanê şari di q1ymet vineni u rocanê bımbarekan dı ınê mewlidi waniyeni.

\section{Noti}

${ }^{\mathbf{1}}$ Ini malumati yo b1 yo Mela Kamilê Pueği ra geriyayi.

\section{Çımeyi}

Babıj, Osman Efendi (1933). Biyişa Pêxımberi, Şam: Hawar Yayınlar1.

Babij, Milla Cima (2009). Sîyerê Neb̂̂, İstanbul: Nûbihar Yayınları.

Beki, Mela Aziz (2015). Diwon Cebexçuri (Ed. Murat Varol), İstanbul: Bingöl Üniversitesi Yayınları.
Canşad, Murad
(2011). Mewlidê
Kirmanckî. www.zazaki.net, (Tarix: 17.11.2016) http://www.zazaki.net/yazi/mewlid-kirmanck-136.htm

Çolîg, Bîlal-Feqî (2012). Mewlîdê Peximbêr Qey Tutonê Zazon, İstanbul: Diwan Yayınları.

Çolîq, Feqî (2016). Dîwonê Baba Tahirê Uryan'î. İstanbul: Astun Neşriyat.
Çolîq, Feqî (2016). Dîwonê Îmamê Şafî̀. İstanbul: Astun Neşriyat.

Demir, Mehmet Akif (2017). Mewlûda Zazakî, İstanbul: Banga Heq Yayınları.

Hunî, Mela Mehemed Elî (2014). Mewlid. İstanbul: Vate Yayınları.

Kavarî, Mela Mehamed (2004). Dîwanê Muhammedê Kavarî. Diyarbakır.

Merdimîn, W.K. (2002). Mela Kamilê Puexî ra Şî̂rî. Vate17, r.69-75.

Merdimîn, W.K. (2002). Werrekîna Inî Melay Mumtazî Biûmênî Yew Ca. Vate-18, r.82-94.

Merdimîn, W.K., Celalî, N. (2003). Ez Ina Zê Yew Mûsîbetêk Vînena. Vate-21, r.4-17.

Muradan, Mela Mehemed (2003). Mewlidî Zazakî. Vate-19, r.18-32.

Muşeki, Mela Abdulqadir (2016). Mewlidê Nebi. Vir-2, r. 730 .

Özel, Ahmet (2004). Mevlid. DİA, 29, Ankara: Türkiye Diyanet Vakfı Yayınları.

Pala, İskender (1999). Ansiklopedik Divan Şiiri Sözlüğü, İstanbul: Ötüken Yayınları.

Puexi, Mela Kamil (2003). Mewlıdi Nebi, İstanbul.

Puexî, Mela Kamil (2003). Mewlidî Nebî. Vate-19, r.76-82.

Xasi, Mela Ehmed (1899). Mewlid-i Nebiyyi El-Qureyşi. Diyarbakır: Litografya Matbaası. 